- NUREG/CR-1515

BNL-NUREG-51221

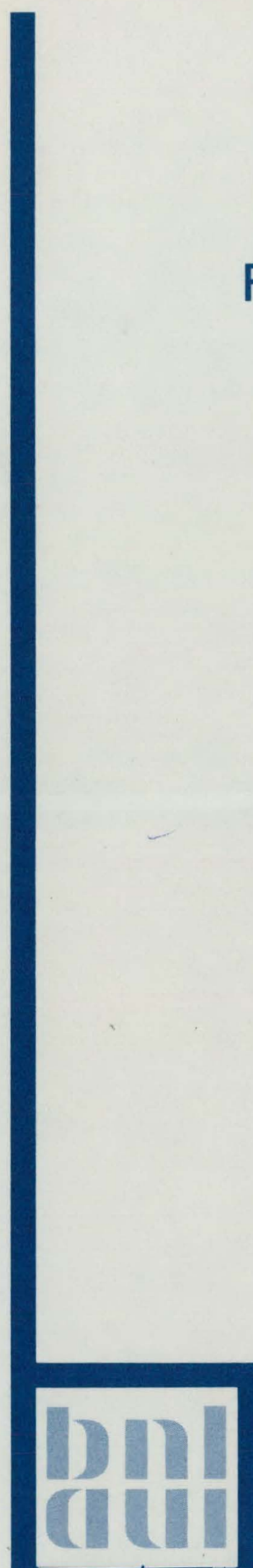

\title{
MASTER
}

\section{FLASHING INCEPTION IN FLOWING LIQUIDS}

OWEN C. JONES, JR.

Date Published: April 1980

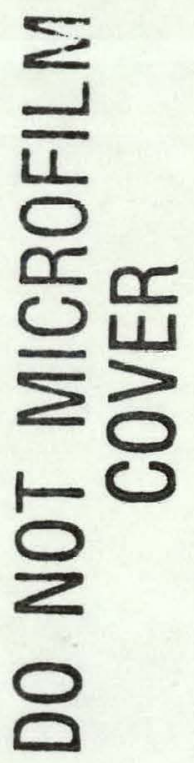

Prepared for

Division of Reactor Safety Research

U.S. Nuclear Regulatory Commission

Office of Nuclear Regulatory Research

Contract No. DE-ACO2-76CHOOO16 


\section{DISCLAIMER}

This report was prepared as an account of work sponsored by an agency of the United States Government. Neither the United States Government nor any agency Thereof, nor any of their employees, makes any warranty, express or implied, or assumes any legal liability or responsibility for the accuracy, completeness, or usefulness of any information, apparatus, product, or process disclosed, or represents that its use would not infringe privately owned rights. Reference herein to any specific commercial product, process, or service by trade name, trademark, manufacturer, or otherwise does not necessarily constitute or imply its endorsement, recommendation, or favoring by the United States Government or any agency thereof. The views and opinions of authors expressed herein do not necessarily state or reflect those of the United States Government or any agency thereof. 


\section{DISCLAIMER}

Portions of this document may be illegible in electronic image products. Images are produced from the best available original document. 
- NUREG/CR-1515

BNL-NUREG-51221

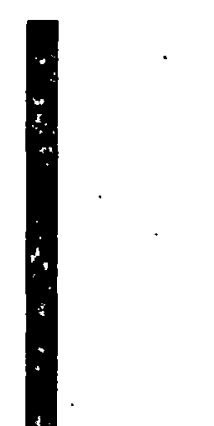

\section{MASTER}

\section{FLASHING INCEPTION IN FLOWING LIQUIDS}

Date Published: April 1980 


\title{
FLASHING INCEPTION IN FLOWING LIQUIDS
}

\author{
by \\ OWEN C. JONES, JR. \\ Department of Nuclear Energy \\ Brookhaven National Laboratory \\ Upton, New York 11973
}

April 1980

PREPARED FOR THE U.S. REGULATORY COMMISSION

OFFICE OF NUCLEAR REGULATORY RESEARCH

CONTRACT NO. DE-AC02-76CH00016

NRC FIN NO. A-3045 


\section{NOTICE}

This report was prepared as an account of work sponsored by an agency of the United States Government. Neither the United States Government nor any agency thereof, or any of their employees, makes any warranty, expressed or implied, or assumes any legal liability or responsibility for any third party's use, or the results of such use, of any information, apparatus, product or process disclosed in this report, or represents that its use by such third party would not infringe privately owned rights.

The views expressed in this report are not necessarily those of the U.S. Nuclear Regulatory Commission.

Available from GPQ Sales Program

Division of Technical Information and Document Control

U.S. Nuclear Regulatory Ciommission

Washington, D.C. 20555 and

National Technical Information Service

Springfield, Virginia 22161 
TABLE OF CONTENTS

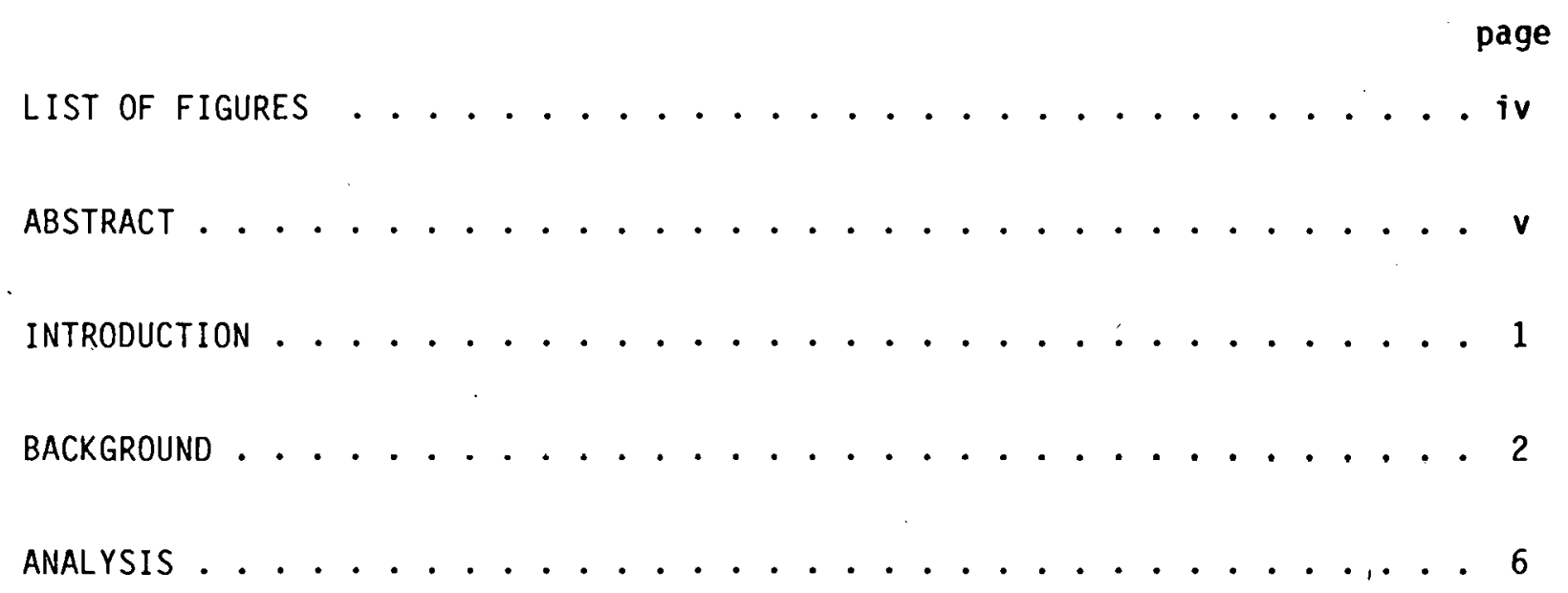

RESULTS AND DISCUSSION . . . . . . . . . . . . . . . . . . . . 11

CONCLUSIONS AND RECOMMENDATIONS . . . . . . . . . . . . . . . . . . 21

ACKNOWLEDGEMENTS . . . . . . . . . . . . . . . . . . . . . . . 22

NOMENCLATURE . . . . . . . . . . . . . . . . . . . . . . . 23

REFERENCES . . . . . . . . . . . . . . . . . . . . . . . 24 
No.

1 observed mass flux effect on overexpansion ............ 3 (underpressure) at flashing inception for the data of Reocreux. [3]

2 Sketch of pressure fluctuation envelope with . . . . . . . . 8 varying mass flux.

3 Qualitative Effects of pressure fluctuations on . . . . . . . 8 observed overexpansion at flashing inception.

4 Velocity and scaled kinetic energy fluctuation . . . . . . . . . 12 intensities calculated from the overexpansion data of Reocreux [3] at flashing inception.

5 Dimensionless correlation of Reocreux's [3] . . . . . . . . 13 overexpansion data at flashing inception.

6 Comparison of the flashing inception data of ........... 14 Reocreux โ3」 and of Seynhaeve, Giot, and Fritte L5] with the theory developed herein using the approximate static flashing overexpansion value of $18 \mathrm{kPa}$ for the computation.

7 Physical combination of the static flashing . . . . . . . . . 16 inception correlation of Alamgir and Lienhard [9] with the flowing turbulence effects described herein. Light lines: static decompression effects only. Dark lines: turbulence effects included.

8 Dimensionless flashing inception pressure . . . . . . . . . . . 19 inception undershoot correlation combining Alamgir and Lienhard $[9\rfloor$ with the turbulence effects developed herein. 


\section{ABSTRACT}

The inception of net vaporization in flashing flows is examined. It is suggested that the flashing inception can be expressed as two additive effects. One is due to the static decompression which is a function of the initial temperature and also of the expansion rate. The other effect which is a function of Reynolds number and flashing index, is due to the turbulent fluctuations of the flowing liquid. It is shown that by taking a three standard deviation band on the turbulent velocity fluctuations, an adequate representation of the inverse mass flux effect on flashing inception for existing data is obtained. The turbulence effects are combined with the correlation of Alamgir and Lienhard to provide predictive methods recommended for the case where both static and convective decompression effects exist. 


\section{INTRODUCTION}

In the event of a leak in a hot high pressure water system, the subcooled liquid will undergo decompression as it flows from the system to the surroundings. This may be a geometrically controlled decompression such as in the case of leakage through a relief valve subsystem. On the other hand, the decompression may be uncontrolled as in the case of a system rupture. In many cases subcooled water flowing towards the leak experiences decompression to pressures below the saturation pressure and at some lower pressure begins to flash to vapor. Flashing may occur in regions of constant cross section such as in pipes, or in regions of varying geometry such as valves, fittings, or regions of a break in the piping system, itself. In cases of concern from a safety standpoint, the resulting two-phase mixture will experience flow limitations due to choking. The actual discharge flow rate, which is especially sensitive to the vapor content, directly affects the system response. This is especially true in nuclear steam supply systems where the heat transfer characteristics of the fuel are quite sensitive to the system liquid inventory. At present there is no general model for the vapor generation rate under nonequilibrium conditions, or for any of the three major factors which affect the void development under such conditions: flashing inception point, interfacial area available for vaporization, and rate of mass exchange per unit interfacial area. It is the purpose of this report to address the question of flashing inception as affected by velocity effects. It will be shown that a model based on turbulent fluctuation intensity does a reasonably acceptable job of accounting for these effects when appljed to the few data avallable. 
BACKGROUND

It is well known that the mass flow rates in critical flow conditions are highly dependent on the vapor content of the flow. Saha][1] has reviewed and evaluated critical flow research concluding that currently accepted equilibrium models underpredict critical flows for "short" pipes especially for subcooled or nearly saturated sources. While thermal nonequilibrium must be taken into account for "short" pipes, it is not clear how the combination of length and diameter enters the picture. Wu et al.[2] have shown that a model based on spherical bubble growth in fields of variable superheat adequately predicts the data of Reocreux[3] for void fractions less than 0.3 . These predictions require accurate knowledge of both the voiding inception point and an initial nucleation density parameter. It is well known[4] that the initial degree of superheat markedly affects bubble growth in both constant and variable pressure fields. The degree of superheat has also been shown to play a strong role in void development in flashing critical flows[2]. Since the point at which flashing inception occurs directly affects the initial superheat, the flashing inception can also be expected to play a strong role in the critical mass flow rates under flashing conditions.

Little work has been accomplished examining the point of flashing inception. Seynhaeve, Giot, and Fritte ${ }^{[5]}$ ran experiments with inlet temperatures between $111^{\circ} \mathrm{C}$ and $167^{\circ} \mathrm{C}$ and at mass fluxes between 10 and $20 \mathrm{Mg} / \mathrm{m}^{2} \cdot \mathrm{sec}$. They determined the superheat at flashing inception to behave inversely with mass flux. Although their data were quite scattered, the superheat apparently decreased to almost zero at the higher mass fluxes, and even became negative in a few cases. In their evaluation of Reocreux's 1.74 bar data, wu et al. [2] found similar 
results as shown in Figure 1. In this figure the superheat is expressed in terms of the overexpansion at the inception point denoted by $\Delta \mathrm{P}_{\mathrm{F}}$. (Note that throughout this report the terms overexpansion and superheat are used interchangeably and are, of course, coupled along the saturation line.) Unfortunately, no other experiment appears to have been heretofore undertaken allowing suitable definition for determination of flashing inception superheats.

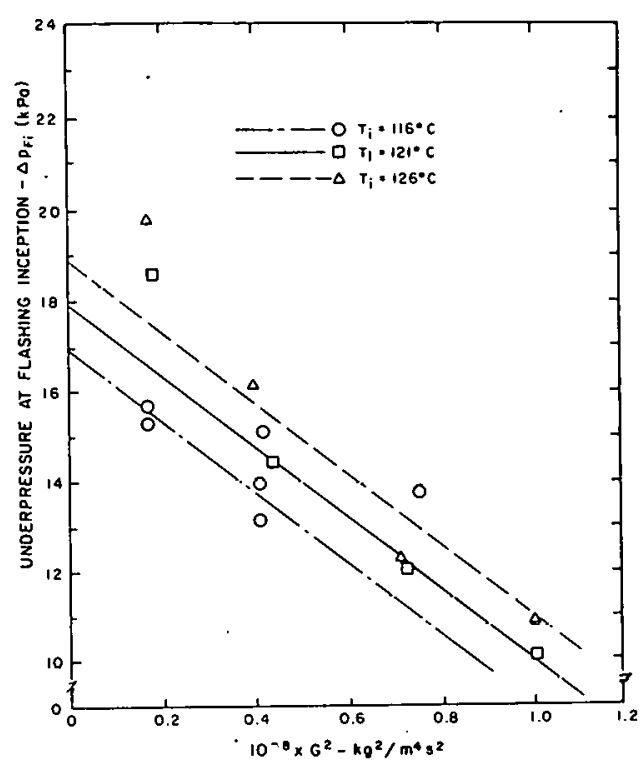

Figure 1. Observed mass flux effect on overexpansion at flashing for the data or Reocreux[3].

(BNL Neg. No. 3-237-79)

The boiling inception and onset of net vapor generation in flowing liquids has undergone much scrutiny in the case of heating, having been the subject of such well-known works as those of $\mathrm{Hsu}^{[6]}$, and of Saha and Zuber.[7], among others. Unfortunately, flashing inception does not appear to be characterized by models applicable to heated liquids where the superheat is generally confined to the wall layer in bulk subcooled liquids. Instead, bulk superheating occurs prior to flashing inception while the initial voiding still seems generally relegated to the wall layer.

In the case of static liquids undergoing rapid decompression, the work of Lienhard, Alamgir, and Trela[8], and of Alamgir and Lienhard[9] are clearly applicable for predicting the flashing inception superheat in nonflowing systems. The former work identified the limit of overexpansion to be dependent on 
the fluid expansion rate (rate of decompression) prior to nucleation up to a value of about $65 \%$ of the spinodal limit. This limit itself depends on the initial temperature. A correlation based on a wide range of data was proposed $[9]$ for the underpressure in the form

$$
\Delta \mathrm{p}_{\text {Fio }}=\Delta \mathrm{p}_{\text {Fio }}{ }^{\circ} \sqrt{1+13.25 \Sigma^{\prime 0.8}}
$$

where the expansion rate was given in units of Matm/sec. The limiting value at vanishing decompression rates was given as a constant dependent on the initial temperature of the fluid only in the form

$$
\Delta \mathrm{p}_{\text {rio }}{ }^{\circ}=0.258 \frac{\sigma^{3 / 2}}{\sqrt{\mathrm{kT}_{\mathrm{c}}}}\left(\frac{\mathrm{T}_{\mathrm{r}}^{13.76}}{1-\frac{\mathrm{v}_{\mathrm{r}}}{\mathrm{v}_{\mathrm{H}}}}\right)
$$

where $\sigma$ is the surface tension, $T_{C}$ the critical tomperature, $T_{r}$ the initial reduced temperature, $v_{f}$ and $v_{g}$ the saturation specific volumes of the liquid and vapor respectively at $T_{r}$, and $k$ is Boltzman's constant. This correlation predicted the static undershoot to an RMS accuracy of $10.6 \%$. in the ranges $0.515<\mathrm{T}_{r}<0.935$ and $0.004<\Sigma^{\prime}<1.803 \mathrm{Matm} / \mathrm{sec}$. A suggestion was made that imperfections in cleaning and preparation of a given system as well as history of preparation may be important. However, this appears unlikely at least within their data scatter since several different systems were indeed correlated.

The superheats predicted by Alamgir and Lienhard $[9]$ range up to $50^{\circ} \mathrm{C}$ or 
more at the highest expansion rates and initial temperatures. Under such severe nonequilibrium conditions, the actual vapor content can be substantially reduced below equilibrium values and actual flow rates very much larger than those predicted on the basis of an equilibrium or near equilibrium critical flow model. Even at very $10 \mathrm{w}$ expansion rates, superheats of over $10^{\circ} \mathrm{C}$ are predicted and observed in fact. The existance of such superheats at flashing inception can be a major factor in inaccurate prediction of two-phase critical flow rates with subcooled initial (or inlet) conditions.

Another major factor identified in the correlation of Reference [9] is the direct behavior of inception superheat predicted with increasing expansion rate. This trend, although in agreement with normally expected homogeneous nucleation behavior, is in direct opposition with the flowing data shown in Figure 1, and with the data of Reference [5]. Unfortunately, however, the decompression rates in these latter experiments were approximately three orders of magnitude slower than those upon which the correlation of $\lfloor 9\rfloor$ is based.

Decompression times in the static systems of References [8] and [9] data are generally less than a millisecond. Decompression times in the flowing systems of [3] and [5] range up to several tens or hundreds of milliseconds. The only other differences hetween the static and dynamic flashing systems seem to be those of fluid motion. Of the factors influenced by these motions; the turbulent pressure fluctuations appear to be those most likely to have an effect.

It thus seems that decompressive flashing inception might be characterized by at least.three considerations: initial temperature; decompression rate; degree of liquid turbulence. Based on these considerations, the balance of this 
report will be devoted to attempting to characterize flashing inception in flowing systems to the extent possible in view of the limited data available. Indeed, it will be shown that the inverse mass flux effects of both References [3] and [5] may be explained by effects of turbulent fluctuations.

ANALYSIS

In view of the preceding remarks, it appears that the condition of the fluid at the onset of flashing, either static or flowing, might be characterized in terms of the turbulent pressure fluctuations. In the static systems of References [8] and [9], it is not likely that turbulence had time to develop. In the flowing systems of References $\lfloor 3\rfloor$ and $\lfloor 5\rfloor$, fully developed turbulence was most certainly present at the high Reynolds numbers encountered lorder of $10^{5}$ ). The following hypotheses thus seem reasonable:

1. Static flashing overexpansion is a function only

of initial temperature and expansion rate, and represents the true inception potential according to Equation (1);

2. Dynamic flashing overexpansion at inception is subject to the additive effects of turbulence giving an apparent alteration in the inception potential. 
It is suggested that the overexpansion at flashing inception may be expressed as

$$
\Delta \mathrm{p}_{\mathrm{Yi}}=f\left[\Delta_{\mathrm{p}_{\mathrm{Fio}}}\left(\mathrm{T}_{\mathrm{i}}, \Sigma^{\prime}\right), \mathrm{p}^{\prime}\right]
$$

where $\Delta P_{F i o}\left(T_{j}, \Sigma^{\prime}\right)$ is the overexpansion under zero flow conditions as dependent on initial temperature, $T_{j}$, and expansion rate, $\Sigma^{\prime}$, and $p^{\prime}$ is the pressure fluctuation.

To see how the pressure fluctuations might enter into the picture, the fluctuation envelope may be envisioned as sketched in Figure 2 depending on the mass flux. As the flux increases, so does the turbulence intensity. According to the hypotheses, the bottom of the envelope would represent the true minimum pressure at any mass flux. If this minimum pressure is taken as identical to the static value at inception represented by hypothesis (1), then the average pressure at the inception point would have to increase with increasing mass tiux as shown in Figure 3 . Since the overexpansion at inception is the difference between the saturation pressure and the observed average pressure, this value, $\triangle P_{F i}$, is seen to decrease in arrordance with observation. If we define the apparcnt overexpansion at flashing inception as

$$
\Delta p_{\mathrm{Fi}}=p_{\mathrm{s}}-\overline{p_{\mathrm{Fi}}}
$$




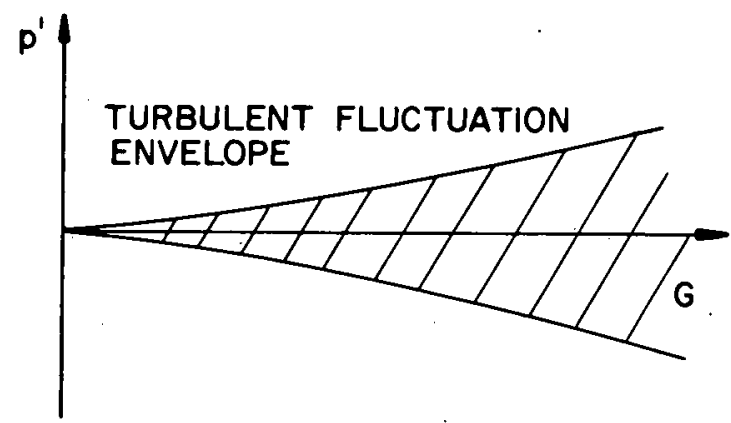

Figure 2. Sketch of pressure fluctuation envelope with varying mass flux. (BNL Neg. No. 3-239-79)

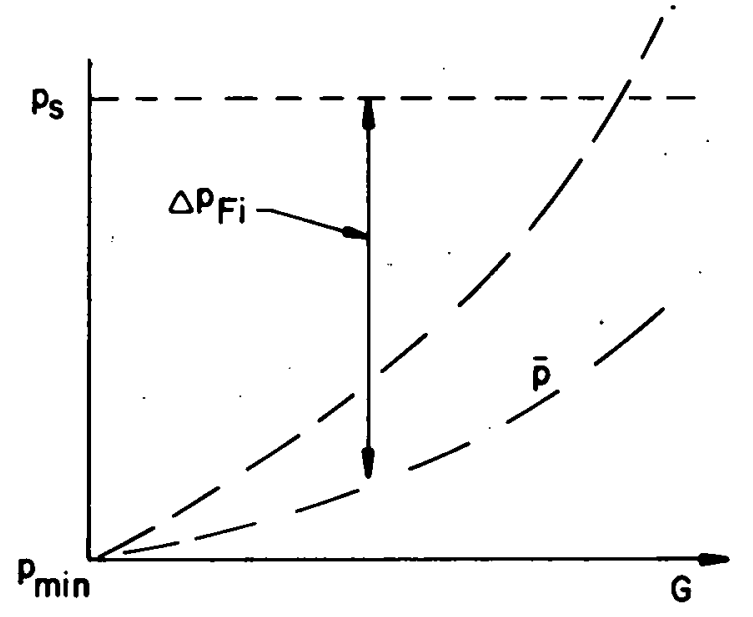

Figure 3. Qualitative effects of pressure fluctuations on observed overexpansion at flashing inception. (BNL Neg. No. 3-239-78)

and the true value is taken to be identical with the static value as

$$
\Delta p_{\text {Fio }}=p_{s}-p_{\min } .
$$

then the relationship between the two, in view of Figures 2 and 3 is simply

$$
\Delta p_{\text {li }}=\Delta p_{\text {Fio }}-\|\left.\mathrm{ax}\right|_{p^{\prime}}
$$


Note that the importance of turbulent pressure fluctuations in cavitation has been previously recognized by Daily and Johnson[10]. They, in fact, point out that the effects of dissolved gas or preexisting gas nuclei will be to reduce the cavitation or flashing inception superheat.

The maximum in the pressure fluctuation envelope is assumed to coincide with the maximum kinetic fluctuations so that

$$
\operatorname{Max}\left|p^{\prime}\right|=\frac{1}{2} \rho_{\ell}\left(u_{\max }^{\prime 2}+v_{\max }^{\prime 2}+w_{\max }^{\prime 2}\right)
$$

The nucleation density monotonically increases with increasing superheat while the probability density of the kinetic energy fluctuations first increases then decreases. The product of nucleation density and superheat probability density is expected to yield a maximum with increasing superheat. This maximum would probably represent the inception point and is expected to fall within the $99 \%$ probability band. If the maximum fluctuation in each velocity component is assumed to be represented by the three sigma value (three standard deviations), then

$$
\operatorname{Max}\left|p^{\prime}\right|=\frac{1}{2} \rho_{\ell}\left[\left(3 \sqrt{\overline{u^{\prime 2}}}\right)^{2}+\left(3 \sqrt{\sqrt{v^{\prime 2}}}\right)^{2}+\left(3 \sqrt{\overline{w^{\prime 2}}}\right)^{2}\right]
$$

so that, for the case of isotropic turbulence (6) becomes 


$$
\Delta \mathrm{p}_{\mathrm{Fi}}=\Delta \mathrm{p}_{\mathrm{Fio}}-27\left(\overline{\frac{\mathrm{u}^{\prime 2}}{\mathrm{U}^{2}}}\right) \frac{\mathrm{G}^{2}}{2 \rho_{\ell}}
$$

where $U$ is the mean velocity of the flow at the inception location and is expected to be dependent to some extent on Reynolds number.

It is seen from Equation (9) that the apparent flashing inception undershoot decreases from the static value as the mass flux increases. Without considering a possible limit on this decrease due to critical flow conditions, an expected limit is a decrease to vanishingly small superheat where the turbulence effects just balance the expansion rate effects at the given temperature. While there is nothing actually prohibiting the turbulent pressure fluctuations from exceeding the values required to overcome the zero-flow incipient superheat, bubbles thus generated would probably collapse almost immediately in the bulk subcooled liquid unless carried to lower pressure regions before this could happen. In dimensionless terms, then, Equation (9) may thus be written considering the latter expectation as

$$
\Delta \mathrm{p}_{\mathrm{Fi}}{ }^{*}=\operatorname{Max}\left\{\begin{array}{l}
0 \\
1-27\left(\overline{\frac{\mathrm{u}^{\prime 2}}{\mathrm{U}^{2}}}\right) \mathrm{F}_{\mathrm{i}}
\end{array}\right.
$$

where $F_{i}$ is termed the flashing index and is the reciprocal of the well-known cavitation index. Note that the apparent flashing inception undershoot has been rendered dimensionless with respect to the static value, which may be predicted by Equation (1). 
RESULTS AND DISCUSSION

Equation (9) shows that the apparent overexpansion at flashing inception should be linear in the square of the mass flux with an intercept of the static inception value, $\Delta p_{F i o}$. If the ideas previously expressed are at all valid, then using extrapolated values of $\Delta \mathrm{PF}_{\mathrm{F}}$, turbulent fluctuation intensities obtained at known inception points should match those found, for instance by Laufer $[11]$, of $0.07-0.08$.

Figure 1 shows the data of Reocreux [3] which appear to be the only data available where inception points are given or can be determined. It should be noticed that $1^{\circ} \mathrm{C}$ represents approximately $6.5 \mathrm{kPa}$ at these temperatures for water. Stated another way, $1 \mathrm{kPa}$ represents $20.15^{\circ} \mathrm{C}$. It is virtually impossible to determine significant trends within the scatter of the data. For this reason, straight lines were utilized to attempt to extrapolate the three sets of data in Figure 1 to a small mass flux consistent with vanishing turbulence, taken to be zero flow herein. The values of $\Delta p_{F i o}$ thus obtained were $1 \%, 18$, and $19 \mathrm{kPa}$, respectively, representative of actual superheats of approximately $3.5^{\circ} \mathrm{C}$. The Alamgir and Lienhard correlation was not used for this purpose since it was not recommended for decompressinn rates below 4000 bar/sec and the maximum values encountered in Reference 3 were approximately $5 \mathrm{bar} / \mathrm{sec}$. (It may be noted that if the correlation is used for these conditions, predictions of approximately $40 \mathrm{kPa}$, or twice those observed, are obtained.)

Using the values of $\triangle P_{F}$ io obtained from Figure 1, the medn fluctuation intensities may be computed from the data. These are shown in Figure 4, (solid symbols). The average of the velocity fluctuation intensities obtained is 0.072 


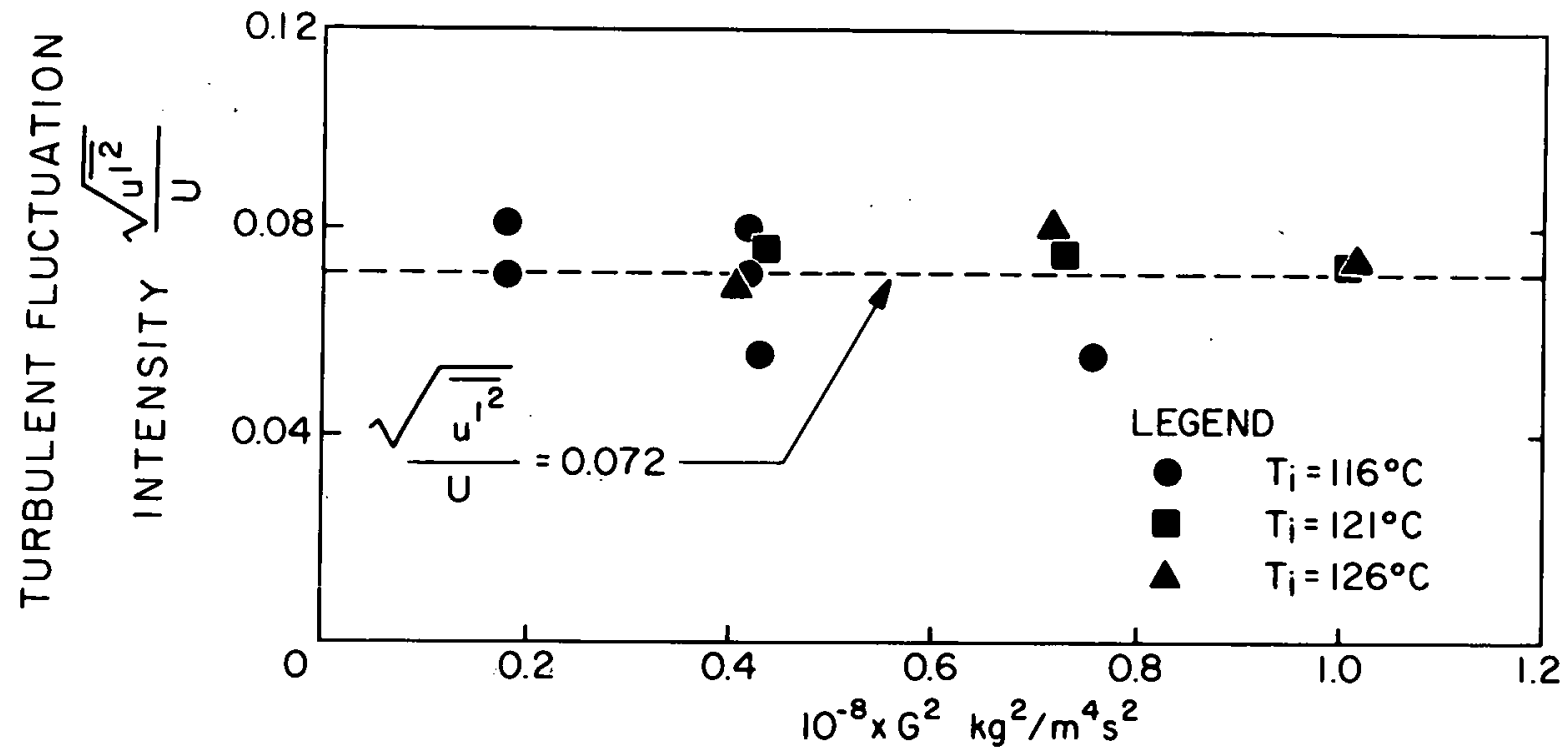

Figure 4. Velocity fluctuation intensities calculated fromn the overexpansion data of Reocreux [3] at flashing inception. (BNL Neg. No. 3-235-79)

in good agreement with the measurements of Daily and Johnson[10] based on their measurements of bubble motion and also of Laufer[11]. No observable trend with mass flux is noticed. Also, there is surprisingly little scatter in the results so obtained.

Reocreux's data are replotted in Figure 5 on dimensionless coordinates suggested by Equation (10). Unfortunately, there are no data with reasonably good values of $\Delta p_{F i o}$ available at higher flashing indices so the equilibrium limit can not be tested.

On the other hand, the data of Seynhaeve, Giot, and Fritte \lfloor\rfloor do consist of higher flashing indices but do not have sufficient information to determine the static inception undershoots. However, these data have expansion rates 
where negligible change from the limiting value $\Delta P_{F}$ io would be expected. Thus, it might be expected that the Reference [5] data in the same temperature range as the Reocreux data may exhibit the same behavior. Both sets of data are plotted in Figure 6. Also. shown in this figure is the prediction based on a static inception underpressure of $18 \mathrm{kPa}$. The trends observed appear to support the conclusions previously stated. Note that the lower limit of zero superheat also appears reasonable and tentatively supported by the relatively meager amount of data available.

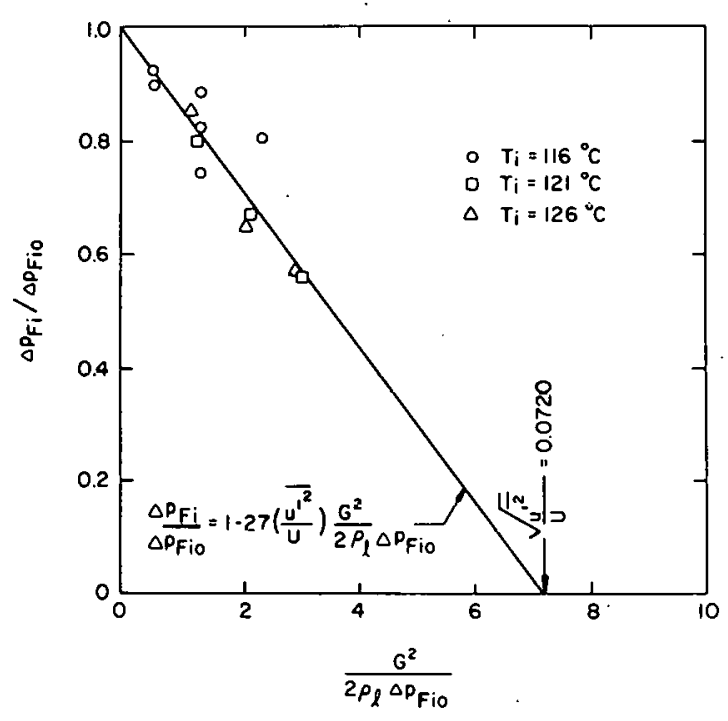

Figure 5. Dimensionless correlation of Reocreux's superheat data at flashing inception.

(BNL Neg. No. 3-240-79)

To provide the reader with some physical insight into the actual behavior of the combined static decompression and flowing effects, the two correlations, that of Reference [9] and that developed herein, may be combined. To demonstrate the effects adequately and simply requires some unfolding of the parameters included in the correlation. A very descriptive and yet simple combination results when the flashing index is written in terms of the expansion rate yielding

$$
\Delta \mathrm{p}_{\mathrm{Fi}}{ }^{+}=\sqrt{1+13.25\left(\Sigma_{\mathrm{o}}^{\prime}+\Delta \Sigma^{\prime}\right)^{0.8}}-\frac{27}{2}\left(\overline{\frac{\mathrm{u}^{\prime 2}}{\mathrm{U}^{2}}}\right) \Phi\left(\Delta \Sigma^{\prime}\right)^{2 / 3}
$$




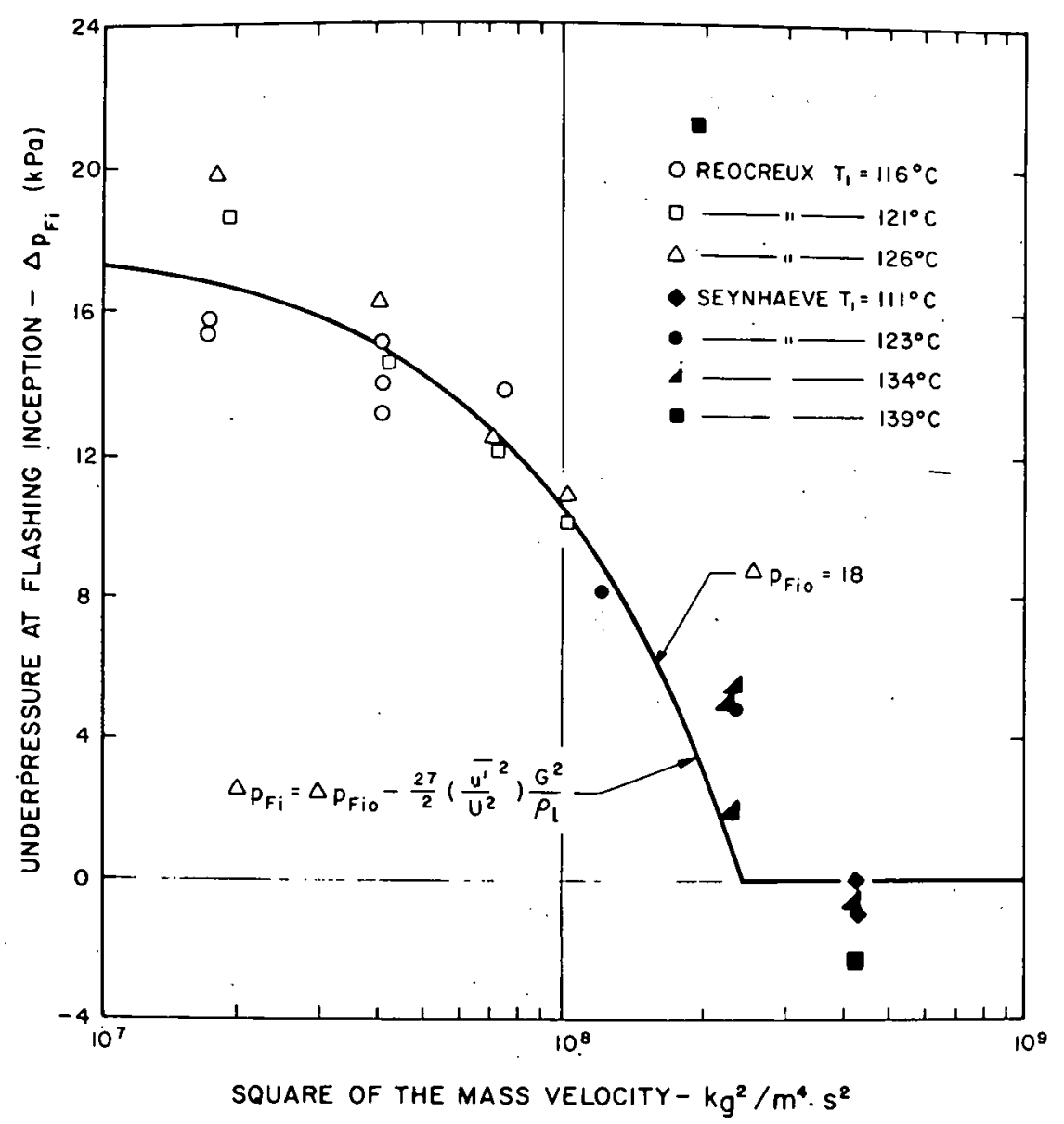

Figure 6. Comparison of the flashing inception data of Reocreux [3] and of Seynhaeve, Giot, and Fritte [5] with the theory developed herein using the approximate static flashing superheat value of $18 \mathrm{kPa}$. for the computation. (BNL Neg. No. 3-238-79)

Note that the total expansion rate, $\Sigma^{\prime}$, has been broken into its static and convective components, $\Sigma_{0}{ }^{\prime}$ and $\Delta \Sigma^{\prime}$, given in the normal Lagrangian manner for a fluid element. Parameters in Equation (11) are seen to be the static and flowing expansion rates and a parameter $\Phi$ given by

$$
\Phi=\frac{\psi}{\Delta \mathrm{p}_{\text {Fio }}} \text {. }
$$


For purposes of this discussion, the turbulence intensity is considered fixed at a value of 0.072 . Indeed, variations from this value as normally found in variable hydrodynamic situations are not expected to be important in view of the stated accuracy of Equation (1).

The parameter $\psi$ is given differently depending on the factor causing the flowing expansion.

$$
\begin{array}{ll}
\text { Friction: } & \psi=\left[2 \mathrm{~d} \sqrt{\rho_{\ell}} / \mathrm{f}\right]^{2 / 3} \\
\text { Acceleration: } & \psi=\left[\mathrm{A} \sqrt{\rho_{\ell}} /(\mathrm{dA} / \mathrm{dz})\right]^{2 / 3}
\end{array}
$$

It should be noted that the streamwise area contraction denoted by $d A / d z$ is also expected to have a significant effect on the local turbulence intensity. This will be discussed in a following report on nozzle flows.

In a physical plane of $\Delta p_{f} j$ vs. $\Sigma^{\prime}$, the static flashing inception $\left(\Delta \Sigma^{\prime}=0\right)$ would appear as a family of curves heginning at constant values, $\Delta p_{F i 0^{\circ}}$, with vanishingly small expansion rates., Then, $\wedge p_{F i}$ increases slowly assymptotic to $\Sigma_{0}{ }^{0.4}$ at high expansion rates. These are shown as the lighter lines at fixed temperature in Figure $7^{\star}$. For practical purposes, $\Delta \mathrm{PFio}^{\circ}$ is limited between 20.2 bar at $100^{\circ} \mathrm{C}$ and 29.5 bar at $\sim 300^{\circ} \mathrm{C}$ after which it decreases again to

*In evaluating Equation (2), surface tension values from the equation given by Schmidt, Properties of Water and Steam in SI-Units, Springer-Verlag, New York, 1969. These values differ from those in the 1979 edition but appear to be more accurate. 


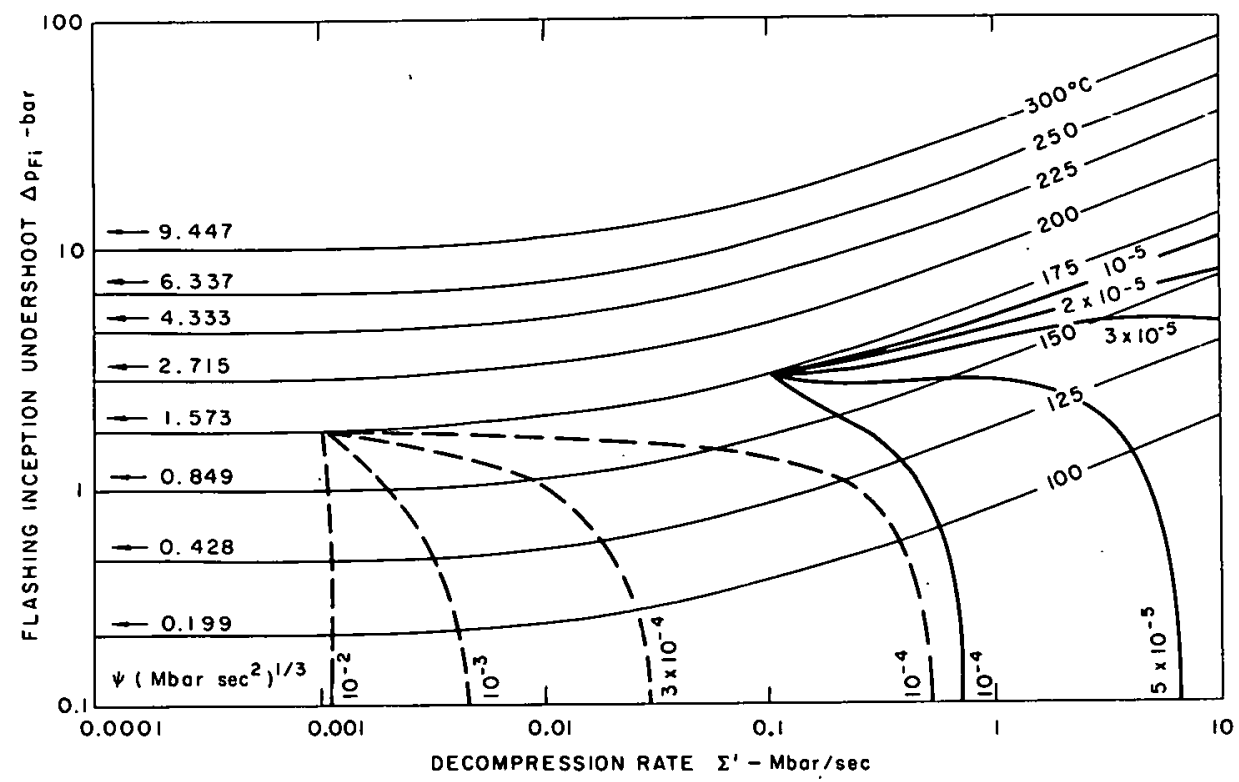

Figure 7 Physical combination of the static flashing inception correlation of Alamgir and Lienhard [9] with the flowing turbulence effects described herein. Light lines: static decompression effects only. Dark lines: turbulence effects included. (BNL Neg. No. 4-367-80)

zero due to vanishing surface tension as the critical point is approached. Less than $15 \%$ increase in inception undershoot is noticed for expansion rates less than $10 \mathrm{kbar} / \mathrm{sec}$ while almost a tenfold increase is predicted at $10 \mathrm{Mbar} / \mathrm{sec}$ expansion rates (beyond the correlation range).

When the convective expansion rate effects are taken into account, four parameters must be considered: $\Delta \mathrm{PF}_{10} 0^{\circ}, \Sigma_{0}{ }^{\prime}, \psi$, and, $\Delta \Sigma^{\prime}$. In this case acurve starting at the static coordinate values of $\left(\Delta \mathrm{PF}_{\mathrm{F}_{0}}, \Sigma_{0}{ }^{\prime}\right)$ departs by increasingly larger amounts from the static curve for increasing total expansion rates depending on the parameter $\psi$. These are the two typical families of lines shown as the dark curves in Figure 7 departing the $175^{\circ} \mathrm{C}$ static decompression curve at values of 0.001 and $0.1 \mathrm{Mbar} / \mathrm{sec}$ for $\Sigma_{0}{ }^{\prime}$. Whether this curve first increases, or decreases monotonically, with increasing $\Sigma$ ' depends on the initial 
slope at the departure point. If two given rates of static decompression are sufficientiy small and dominated by large values of convective expansion rates such that a given total value of $\Sigma^{\prime}$ is large with respect to the two static values, curves of the same value of $\psi$ will tend to coalesce. This is seen for the two curves in Figure 7 where $\psi=10^{-4}$. Indeed, for $\Sigma^{\prime}$ greater than approximately 0.1 , the two different curves for $\psi=5 \times 10^{-5}$ are virtually indistinguishable.

The dimensionless plane suggested by Equation (11) reduces the complexity by one dimension while still keeping the general pictorial behavior of the phenomenon unchanged. By plotting the dimensionless undershoot, $\Delta \mathrm{p}_{\mathrm{F}}{ }^{+}$, as a function of the convective expansion rate, $\Delta \Sigma^{\prime}$, (Figure 8 ), the essence of the physical behavior is maintained while providing a much simplified picture. It is noted that the expected range of $\psi$ for friction-caused expansions is $10^{-4}$ to $10^{-2}$ (Mbar $\left.\sec ^{2}\right)^{1 / 3}$ while those for accelerated cases is one to two orders of magnitude smaller for the various nozzles encountered in the critical flow literature. The normal range for $\Phi$ is then seen to be a six-order range between 0.1 and $10^{5}(\mathrm{sec} / \mathrm{Mbar})^{2 / 3}$. In the case of the Reference [3] experiments, $\psi$ is near 0.004 (Mbar $\left.\sec ^{2}\right)^{1 / 3}$ while, based on the extrapolated values of $\Delta p_{F} i_{0}, \Phi$ is near 20,000 (scc/Mbar) $2 / 3$.

As seen in Figure 8 , at a given value of $\Sigma_{0}$ ' the curves become assymptotic at small $\Sigma^{\prime}$ to the ratio of the static value of undershoot to that for vanishing expansion rates at a given temperature. For increasing convective expansions, the undershoot may or may not first increase; depending on $L_{0}^{\prime}$ and $\Phi$, but then decreases rapidly to zero, indicating the disappearance of any significant amounts of nonequilibrium. 
In estimating the degree of nonequilibrium superheat to be expected at the onset of flashing in constant area ducts 1 a following report will describe application to variable area geometries), it seems easiest to utilize Equations (1) (2), and (10) with the turbulence intensity taken as 0.072 (unless a clear and substantially different value is known a priori for a particular geometry). In practice, the light lines in Figure 7 can probably be used to estimate $\triangle P_{F}$ io with sufficient accuracy in many cases so that Equations (1) and (2) need not be used. On the other hand, conditions near atmospheric pressure similar to those of References (3) and (5) where expansions on the order of $20 x$ $10^{-6} \mathrm{Mbar} / \mathrm{sec}(20 \mathrm{bar} / \mathrm{sec})$ and less were encountered, it appears that the static inception criteria (Ref [9]) may be considerably in error if extrapolated below its range of applicability. In such cases, independent means of developing static inception estimates do not exist and alternate methods are required. 


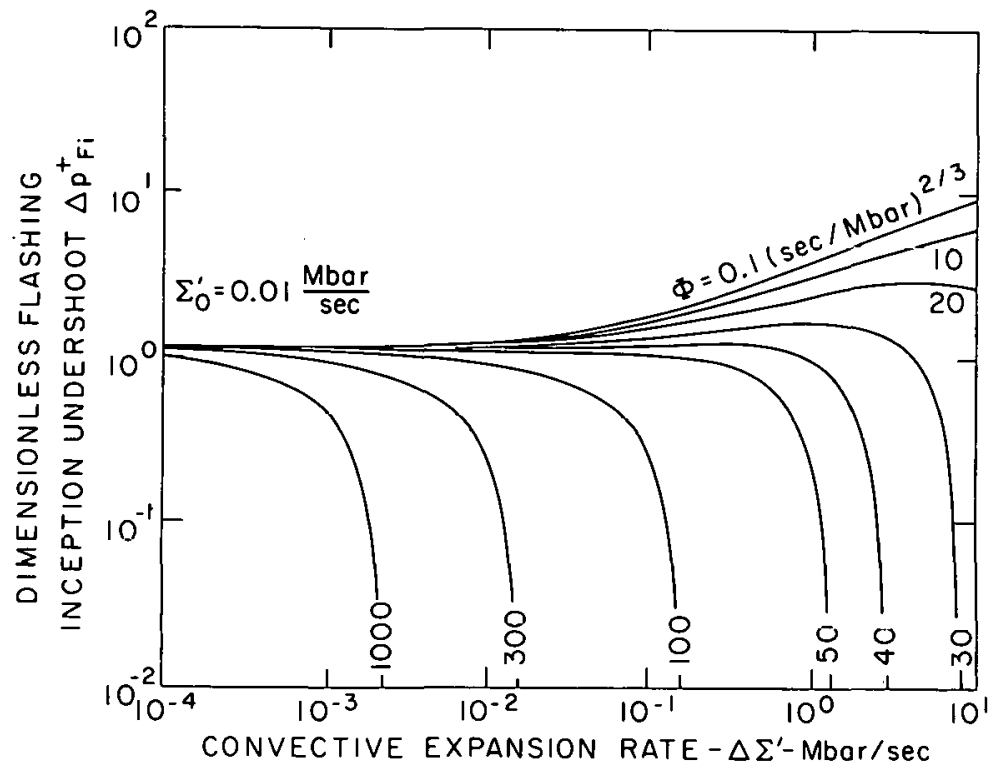

(a) $0.01 \mathrm{Mbar} / \mathrm{sec}$ convective expansion rate (BNL Neg. No. 4-368-80)

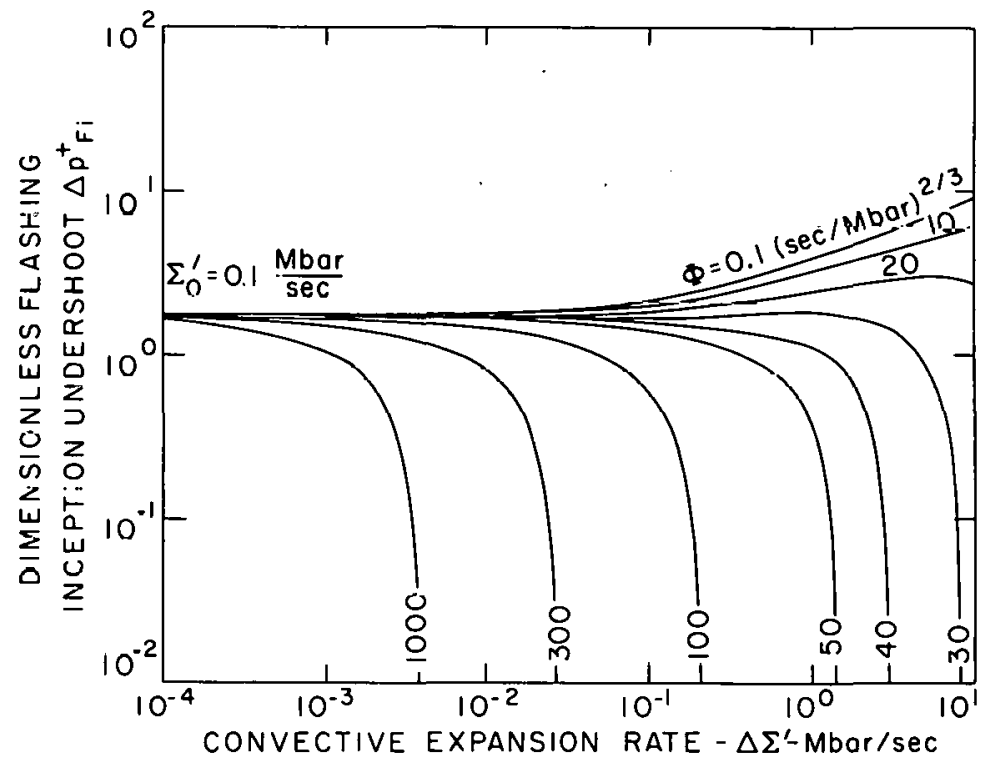

(b) $0.1 \mathrm{Mbar} / \mathrm{sec}$ convective expansion rate (BNL Neg. No. 4-370-80)

Figure 8 Dimensionless flashing inception pressure inception undershoot correlation combining Alamgir and Lienhard [9] with the turbulence effects developed herein.

(CONTINUED ON NEXT PAGE) 


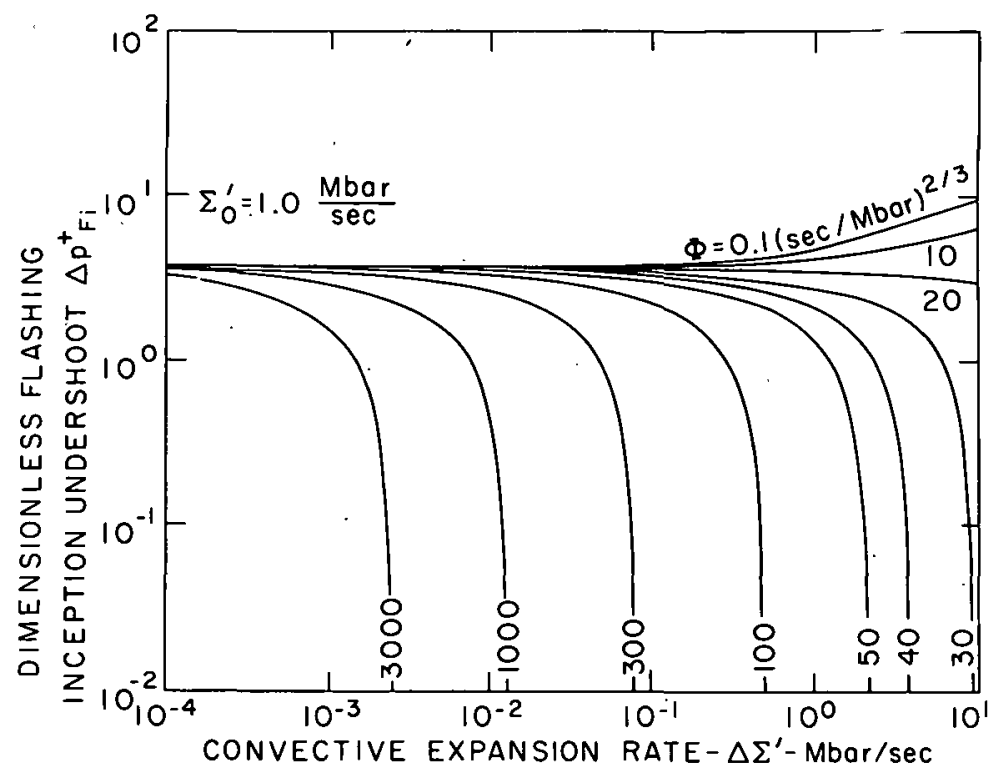

(c) $1.0 \mathrm{Mbar} / \mathrm{sec}$ convective expansion rate (BNL Neg. No. 4-369-80)

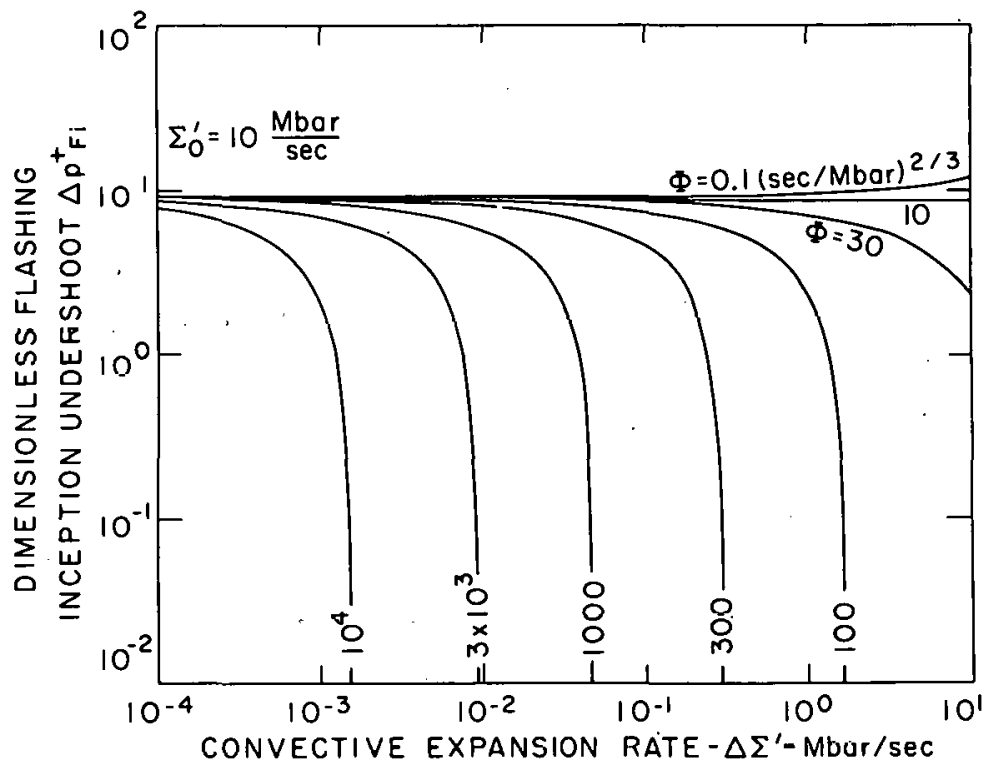

(d) $10.0 \mathrm{Mbar} / \mathrm{sec}$ convective expansion rate (BNL Neg. No. 4-371-80)

Figure 8 Dimensionless flashing inception pressure inception undershoot correlation combining Alamgir and Lienhard [9] with the turbulence effects developed herein.

(CONTINUED FROM PREVIOUS PAGE) 
CONCLUSIONS AND RECOMMENDATIONS

1. Flashing inception superheat in flowing systems appears to be described by the effects of turbulent fluctuations (Equation 10). This suggests that the flowing and static superheats at inception are identical once turbulent fluctuations are taken into account.

2. Under flowing conditions where flashing occurs under the combined effects of system decompression and convective decompression caused by friction and/or acceleration, the flashing inception superheat may be either very large (up to $50^{\circ} \mathrm{C}$ or more) or may instead be negligible. The actual superheat depends on the relative balance between apparent expansion rate and turbulence effects. Since the degree of nonequilibrium depends heavily on the initial superheat at flashing inception, the resultant void development is expected to be strongly affected.

3. It is suggested that since critical flow rates of two-phase mixtures are very dependent on the actual vapor content of the flowing mixture and hence on the void development, that the variations in flashing inception superheat may be of overriding importance in surh flows and, in fact, may be the prime cause of much of the apparent. sratter in the existing data.

4. It is suggested that the limit of flashing inception with vanishing mass flux in flowing systems coincides with that value that would be obtained by static decompression at the same expansion rates. 
5. Methods of accurately extending the static flashing inception superheat correlation of Alamgir and Lienhard [9] to lower values of expansion rate are required.

6. Additional data are needed for flashing inception in flowing systems at higher pressures. Sufficient detail are required to accurately determine the inception point due to the sensitivity of void development to small changes in superheat at inception. Sufficient range in mass flux is required to allow extrapolative determination of the case of vanishing turbulence.

\section{ACKNOWLEDGEMENTS}

The author is grateful for numerous enlightening discussions with Prof. J.H. Lienhard, and Drs. N. Abuaf and B.J.C. Wu. This work was performed under the auspices of the U.S. Nuclear Regulatory Commission. 
NOMENCLATURE

\section{Engl ish}

$A=$ duct cross section area

$d=$ diameter

$f=D^{\prime}$ Arcy friction factor

$F_{j}=$ flashing index

$k=$ Boltzman's constant

$G=\operatorname{mass}$ flux

$p=$ pressure

$T=$ temperature

$u^{\prime}, v^{\prime}, w^{\prime}=$ velocity fluctuation components

$U_{0}=$ local channel mass-averaged velocity at inception point

$z=$ streamwise coordinate

\section{Greek}

$\Delta=$ difference

$\Phi=$ parameter defined by Equation (12)

$\mu=$ viscosity

$\rho=$ density

$\sigma=$ surface tension

$\Sigma^{\prime}=$ rate of pressure decrease (expansion rate)

$\Sigma_{0}^{\prime}=$ static expansion rate, $\partial \mathrm{p} / \partial \mathrm{t}$

$\Delta \Sigma=$ convective expansion rate, $v \partial \mathrm{p} / \partial z$

$\psi=$ parameter defined by Equation (13)

\section{Subscripts}

$c=$ critical

$f=$ saturated liquid

$F i=$ flashing inception

$F i o=$ flashing inception under static conditions

$g$ = saturated vapor

$i=$ initial

$\ell=1$ iquid

$\max =\operatorname{maximum}$

min= minimum

$0=$ static value without convective effects

$r=$ reduced minimum

$s$ = saturation value

\section{Superscripts}

$\star,+$ = dimensionless

$\dot{ }=$ fluctuation

- averaged

- = at vanishing expansion rates 


\section{REFERENCES}

[1] P. Saha, "A Review of Two-Phase Steam-Water Critical Flow Models with Emphasis on Thermal Nonequilibrium," BNL-NUREG-50907, September, 1978.

L2」B.J.C. Wu, P. Saha, N. Abuaf, and O.C. Jones, Jr., "A One-Dimensional Model of Vapor Generation in Steady Flashing Flow: Interim Milestone Report," BNL-NUREG-25709, October, 1978.

[3] M. Reocreux, "Contribution a l'Etude des Debits Critiques en Ecoulement Diphasique Eau-Vapeur," Pho Thesis, Universite Scientifique et Medicale de Grenoble, France, 1974.

[4] 0.C. Jones, Jr., and N. Zuber, "Bubble Growth in Variable Pressure Fields," Trans. ASME, J. Heat Trans., 100C, 453-459, 1978.

[5] J.M. Seynhaeve, M.M. Giot, and A.A. Fritte, "Non-Equilibrium Effects on Critical Flow Rates at Low Qualities," presented at the Specialists Meeting on Transient Two-Phase Flow--Toronto, Canada, August 3-4, 1976.

[6] Y.Y. Hsu, "On the Size Range of Active Nucleation Cavities on a Heating Surface," Trans. ASME, J. Heat Trans., 84C, 207-216, 1962.

[7] P. Saha, and N. Zuber, "Point of Net Vapor Generation and Vapor Void Fraction in Subcooled Boiling," in Heat Transfer 1974, Proc 5th Int. Heat Trans. Conf., Vol. IV, 175-1/9, 1974. 
L8」 J.H. Lienhard, Md. Alamgir, and M. Trela, "Early Response of Hot Water to Sudden Release from High Pressure," Trans. ASME, J. Heat Trans., 100C, 473-479, 1978.

[9」 Alamgir, Md., and Lienhard, J.H., "Correlation of Pressure Undershoot During Hot-Water Depressurization," Trans ASME, J. Heat Trans., in press.

[10] J.W. Daily, and V.E. Johnson, Jr., "Turbulence and Boundary Layer Effects on Cavitation Inception from Gas Nuclei," Trans. ASME, 78 1695-1706, 1956.

[11] J. Laufer, "Investigation of Turbulent Flow in a Two-Dimensional Channel," NACA TN 2123, July, 1950.

$-25-$ 